\title{
Interval Estimation for Ratios of Correlated Age-Adjusted Rates
}

\author{
Ram C. Tiwari ${ }^{1}$, Yi Li ${ }^{2}$ and Zhaohui $\mathrm{Zou}^{3}$ \\ ${ }^{1}$ Food and Drug Administration, ${ }^{2}$ Harvard School of Public Health and \\ ${ }^{3}$ Information Management Services
}

\begin{abstract}
Providing reliable estimates of the ratios of cancer incidence and mortality rates across geographic regions has been important for the National cancer Institute (NCI) Surveillance, Epidemiology, and End Results (SEER) Program as it profiles cancer risk factors as well decides cancer control planning. A fundamental difficulty, however, arises when such ratios have to be computed to compare the rate of a subregion (e.g., California) with that of a parent region (e.g., the US). Such a comparison is often made for policy-making purposes. Based on F-approximations as well as normal approximations, this paper provides new confidence intervals (CIs) for such rate ratios. Intensive simulations, which capture the real issues with the observed mortality data, reveal that these two CIs perform well. In general, for rare cancer sites, the $F$-intervals are often more conservative, and for moderate and common cancers, all intervals perform similarly.
\end{abstract}

Key words: Cancer rate-ratio, F-approximation, normal-approximation, 2000 US standards.

\section{Introduction and Preliminaries}

Let $\Omega$ denote a region such as the entire US or a state in the US, and let $X$ denote a subregion (a proper subset) of $\Omega$. Denote the rest of the region by $X^{c}=\Omega / X$. Let $R_{X}, R_{X^{c}}$ and $R_{\Omega}$ denote the age-adjusted rates for $X, X^{c}$ and $\Omega$, respectively, all of which are defined below.

$$
R_{X}=\sum_{j=1}^{J} w_{j} \frac{d_{X j}}{n_{X j}}, R_{X^{c}}=\sum_{j=1}^{J} w_{j} \frac{d_{X^{c} j}}{n_{X^{c} j}}, R_{\Omega}=\sum_{j=1}^{J} w_{j} \frac{d_{\Omega j}}{n_{\Omega j}},
$$

where $w_{j}$ are known standards normalized to sum to 1 over the $J$ age-groups; $d_{X j}, d_{X^{c} j}, d_{\Omega j}$ and $n_{X j}, n_{X^{c} j}, n_{\Omega j}$ are the number of cancer cases or deaths, and the number of person-years in $X, X^{c}, \Omega$, respectively.

We define the underlying true rates as $\mu_{X}=E\left(R_{X}\right), \mu_{X^{c}}=E\left(R_{X^{c}}\right), \mu_{\Omega}=$ $E\left(R_{\Omega}\right)$, whose unbiased point estimates are $\widehat{\mu_{X}}=R_{X}, \widehat{\mu_{X^{c}}}=R_{X^{c}}$ and $\widehat{\mu_{\Omega}}=R_{\Omega}$, 
respectively. Under the assumption that the counts are independent Poisson random variables, their variances can be approximated by

$$
V_{X} \cong \sum_{j=1}^{J} w_{j}^{2} \frac{d_{X j}}{n_{X j}^{2}}, V_{X^{c}} \cong \sum_{j=1}^{J} w_{j}^{2} \frac{d_{X^{c} j}}{n_{X^{c} j}^{2}}, V_{\Omega} \cong \sum_{j=1}^{J} w_{j}^{2} \frac{d_{\Omega j}}{n_{\Omega j}^{2}}
$$

Our interest is to construct an approximate $100(1-\alpha) \%$ confidence interval for the rate-ratio of $X$ to $\Omega$, namely that of the parameter $\theta=\frac{\mu_{X}}{\mu_{\Omega}}$, e.g the cancer rate-ratio comparing the rate of California with that of the US. Such comparisons are often necessary for policy-making purposes. However, statistical diffculties arise when the estimates of the rates (e.g. those of California and the US) are correlated.

In a simpler context, Fay (1999) and Tiwari et al. (2006) derived confidence intervals for $\phi=\frac{\mu_{X}}{\mu_{X^{c}}}$ using the $F$ distribution as an approximation of the ratio of the two independent Gamma random variables. Specifically, denote by $\hat{\phi}=\frac{R_{X}}{R_{X^{c}}}$, the estimator of $\phi$. The F-based confidence interval is given in Tiwari et al. (2006) as

$$
\left(\frac{R_{X}}{R_{X^{c}}} F_{\left(\left(2 R_{X}^{2}\right) / V_{X},\left(2 R_{X^{c}}^{2} / V_{X^{c}}\right)\right)}^{-1}\left(\frac{\alpha}{2}\right), \frac{R_{X}}{R_{X^{c}}} F_{\left(\left(2 R_{X}^{2}\right) / V_{X},\left(2 R_{X^{c}}^{2}\right) / V_{X^{c}}\right)}^{-1}\left(1-\frac{\alpha}{2}\right)\right),
$$

where $F_{(a, b)}^{-1}(p)$ is the $p^{t h}$ percentile of $F_{(a, b)}$. The derivation of (1.1) is to view the ratio $\hat{\phi}$ as an approximately $F$ distributed random variable, given the independence of the numerator and the denominator in $\hat{\phi}$.

Tiwari et al. (2006) noted the need for comparing the rate of a subregion (e.g., California) with that of a larger region (e.g., the US), and gave a possible solution for computing the CIs for $\theta$ (the ratio of a subregion with its parent region) that accounts for the correlation between the age-adjusted rates. However, their method was not fully developed. In this paper, we will propose new $F$ - and normal-based confidence intervals that can conveniently compute the CIS based on the ratio of two correlated estimates of rates. We demonstrate via simulations that the new intervals perform well and retain the nominal coverage probabilities.

The rest of the paper is organized as follows. In Section 2, we derive the new confidence intervals, and in Section 3 we evaluate their performance in terms of their empirical coverage probabilities. Section 4 gives a short discussion and Section 5 ends this paper with a conclusion.

\section{Two New Confidence Intervals for $\theta$}

To derive the confidence interval for $\theta$ based on its point estimate $\hat{\theta}$, we first 
assume that

$$
\frac{n_{X 1}}{n_{\Omega 1}} \doteq \frac{n_{X 2}}{n_{\Omega 2}} \doteq \ldots \doteq \frac{n_{X J}}{n_{\Omega J}} \doteq p_{X}
$$

That is, the ratio of person years in $X$ (e.g. California) to that of $\Omega$ (e.g. the US) is approximately the same across all age-groups. This so-called proportional agedistribution assumption is common in comparing the age-adjusted rates across different geographical areas and was found to be a good approximation for the US population; see, e.g., Pickle and White (1995).

Reasonable values for $p_{X}$ is given by $\hat{p}_{X}=\frac{n_{X}}{n_{\Omega}}$, where $n_{X}=\sum_{j} n_{X j}$ and $n_{\Omega}=\sum_{j} n_{\Omega j}$. Now, we can write

$$
\begin{aligned}
R_{\Omega} & =\sum_{j=1}^{J} w_{j} \frac{d_{\Omega j}}{n_{\Omega j}} \\
& =\sum_{j=1}^{J} w_{j} \frac{d_{X j}+d_{X^{c} j}}{n_{\Omega j}} \\
& =\sum_{j=1}^{J} w_{j} \frac{n_{X j}}{n_{\Omega j}}\left(\frac{d_{X j}}{n_{X j}}\right)+\sum_{j=1}^{J} w_{j} \frac{n_{X^{c} j}}{n_{\Omega j}}\left(\frac{d_{X^{c} j}}{n_{X^{c} j}}\right) \\
& \approx p_{X} R_{X}+p_{X^{c}} R_{X^{c}},
\end{aligned}
$$

where $p_{X^{c}}=1-p_{X}$. Write the estimators for $\theta$ and $\phi$ by

$$
\hat{\theta}=\frac{R_{X}}{R_{\Omega}}=\frac{R_{X} / R_{X^{c}}}{\hat{p}_{X}\left(R_{X} / R_{X^{c}}\right)+\hat{p}_{X^{c}}}
$$

and

$$
\hat{\phi}=\frac{R_{X}}{R_{X^{c}}}=\frac{\left(\frac{R_{X}}{R_{\Omega}}\right)\left(1-\hat{p}_{X}\right)}{1-\hat{p}_{X}\left(\frac{R_{X}}{R_{\Omega}}\right)}
$$

respectively, where $\hat{p}_{X^{c}}=1-\hat{p}_{X}$. Also note that

$$
\theta=\frac{\phi}{p_{X} \phi+p_{X^{c}}}
$$

Hence confidence intervals for $\phi$ would lead to those for $\theta$ (and vice versa), which will be derived below. 


\subsection{An $F$-approximation}

Let $\left(\phi_{L(\alpha / 2)}, \phi_{U(\alpha / 2)}\right)$ be the $100(1-\alpha) \% F$ confidence interval for $\phi$ given in (1.1). Hence,

$$
P\left(\phi_{L(\alpha / 2)}<\phi<\phi_{U(\alpha / 2)}\right)=1-\alpha .
$$

Let $g(t)=1-p_{X^{c}} /\left(p_{X} t+p_{X^{c}}\right)$ for short. Note that in this notation, $g(\phi) p_{X}=$ $1-p_{X^{c}} /\left(p_{X} \phi+p_{X^{c}}\right)=\theta$. As $g(\phi)$ is increasing in $\phi,(2.1)$ is equivalent to

$$
P\left(\frac{1}{p_{X}} g\left(\phi_{L(\alpha / 2)}\right)<\theta<\frac{1}{p_{X}} g\left(\phi_{U(\alpha / 2)}\right)\right)=1-\alpha .
$$

This finds the 100(1- $\alpha) \%$ CI for $\phi$.

Since $\hat{p}_{X}$ and $\hat{p}_{X^{c}}$ consistently estimate $p_{X}$ and $p_{X^{c}}$ respectively, we thus obtain the approximate $100(1-\alpha) \%$ confidence interval for $\theta$ as

$$
\left(\frac{\phi_{L(\alpha / 2)}}{\hat{p}_{X} \phi_{L(\alpha / 2)}+\hat{p}_{X^{c}}}, \frac{\phi_{U \alpha / 2)}}{\hat{p}_{X} \phi_{U(\alpha / 2)}+\hat{p}_{X^{c}}}\right) \text {. }
$$

\subsection{A normal approximation}

From the perspective of a normal approximation, we can also derive a confidence interval for $\theta$. First note that

$$
\hat{\theta}=\frac{R_{X}}{\hat{p}_{X} R_{X}+\hat{p}_{X^{c}} R_{X^{c}}}
$$

Using a Taylor series,

$$
\begin{aligned}
& \frac{R_{X}}{\hat{p}_{X} R_{X}+\hat{p}_{X^{c}} R_{X^{c}}} \\
= & f\left(R_{X}, R_{X^{c}}\right) \\
\approx & f\left(\mu_{X}, \mu_{X^{c}}\right)+\left(R_{X}-\mu_{X}\right) f_{X}\left(\mu_{X}, \mu_{X^{c}}\right)+\left(R_{X^{c}}-\mu_{X^{c}}\right) f_{X^{c}}\left(\mu_{X}, \mu_{X^{c}}\right) \\
& f\left(\mu_{X}, \mu_{X^{c}}\right)+\left(R_{X}-\mu_{X}\right)\left(\frac{\hat{p}_{X^{c}} \mu_{X^{c}}}{\left(\hat{p}_{X} \mu_{X}+\hat{p}_{X^{c}} \mu_{X^{c}}\right)^{2}}\right) \\
& +\left(R_{X^{c}}-\mu_{X^{c}}\right)\left(\frac{\hat{p}_{X^{c}} \mu_{X}}{\left(\hat{p}_{X} \mu_{X}+\hat{p}_{X^{c}} \mu_{X^{c}}\right)^{2}}\right)
\end{aligned}
$$


and

$$
\begin{aligned}
\mu_{\theta} & =E\left(\frac{R_{X}}{\hat{p}_{X} R_{X}+\hat{p}_{X^{c}} R_{X^{c}}}\right) \\
& \approx \frac{\mu_{X}}{\hat{p}_{X} \mu_{X}+\hat{p}_{X^{c}} \mu_{X^{c}}} \\
V_{\theta} & =\operatorname{Var}\left(\frac{R_{X}}{\hat{p}_{X} R_{X}+\hat{p}_{X^{c}} R_{X^{c}}}\right) \\
& \cong\left\{\frac{\hat{p}_{X^{c}} \mu_{X^{c}}}{\left(\hat{p}_{X} \mu_{X}+\hat{p}_{X^{c}} \mu_{X^{c}}\right)^{2}}\right\}^{2} V_{X}+\left\{\frac{\hat{p}_{X^{c}} \mu_{X}}{\left(\hat{p}_{X} \mu_{X}+\hat{p}_{X^{c}} \mu_{X^{c}}\right)^{2}}\right\}^{2} V_{X^{c}} \\
& =\left\{\frac{\hat{p}_{X^{c}} \mu_{X^{c}} \mu_{X}}{\left(\hat{p}_{X} \mu_{X}+\hat{p}_{X^{c}} \mu_{X^{c}}\right)^{2}}\right\}^{2}\left(\frac{V_{X}}{\mu_{X}^{2}}+\frac{V_{X^{c}}}{\mu_{X^{c}}^{2}}\right) .
\end{aligned}
$$

Now, since $\left(R_{X}, R_{X^{c}}\right)$ is asymptotically normal, and $\frac{R_{X}}{\hat{p}_{X} R_{X}+\hat{p}_{X^{c}} R_{X^{c}}}=f\left(R_{X}, R_{X^{c}}\right)$ is a continuous function of $\left(R_{X}, R_{X^{c}}\right)$, we have that $\frac{R_{X}}{\hat{p}_{X} R_{X}+\hat{p}_{X^{c}} R_{X^{c}}}$ is asymptotically normal with mean $\mu_{\theta}$ and variance $V_{\theta}$. Hence an approximate $100(1-\alpha) \%$ confidence interval for $\theta \cong \frac{\mu_{X}}{\mu_{\Omega}}$ is given by

$$
\left(\frac{R_{X}}{\hat{p}_{X} R_{X}+\hat{p}_{X^{c}} R_{X^{c}}}-Z_{\alpha / 2} \sqrt{\hat{V}_{\theta}}, \frac{R_{X}}{\hat{p}_{X} R_{X}+\hat{p}_{X^{c}} R_{X^{c}}}-Z_{\alpha / 2} \sqrt{\hat{V}_{\theta}}\right),
$$

where $Z_{\alpha}$ is the upper $100 \alpha$ percentile point of the standard normal distribution, and

$\hat{V}_{\theta}=\left\{\frac{\hat{p}_{X^{c}} R_{X^{c}} R_{X}}{\left(\hat{p}_{X} R_{X}+\hat{p}_{X^{c}} R_{X^{c}}\right)^{2}}\right\}^{2}\left(\frac{V_{X}}{R_{X}^{2}}+\frac{V_{X^{c}}}{R_{X^{c}}}\right) \doteq\left\{\frac{\hat{p}_{X^{c}} R_{X^{c}} R_{X}}{R_{\Omega}^{2}}\right\}^{2}\left(\frac{V_{X}}{R_{X}^{2}}+\frac{V_{X^{c}}}{R_{X^{c}}^{2}}\right)$.

Thus, the normal confidence interval for $\theta$ is

$$
\left(\frac{R_{X}}{R_{\Omega}} \pm Z_{\alpha / 2} \frac{R_{X}}{R_{\Omega}^{2}} \sqrt{\hat{p}_{X^{c}} R_{X^{c}}^{2}\left(\frac{V_{X}}{R_{X}^{2}}+\frac{V_{X^{c}}}{R_{X^{c}}^{2}}\right)}\right) .
$$

\section{Simulation Studies}

We carried out simulations along the lines of Tiwari et al. (2006). We used the 2004 US cancer mortality data for tongue, esophagus, and lung cancer sites. These sites were selected to reflect the spectrum of cancer incidence; that is, from rare cancer (tongue), to moderate cancer (esophagus), to common cancer (lung). 
The data were used to generate Poisson counts $d_{X j}$, where $X$ represents each of the 51 regions (50 states and Washington D.C.) and $j$ indexes the 19 age-groups. The true means of the Poisson distributions are taken to be the observed values of $d_{X j}$. We generated 10,000 Poisson counts, and the computed age-adjusted rates, using the 2000 US standards, so that $\sum_{j=1}^{19} w_{j}=1$. Approximate $95 \%$ confidence intervals were obtained for the ratios of the age-adjusted rates for each of the 51 regions as compared to the overall US rate using the modified versions of the two CIs, as discussed in Tiwari et al. (2006).

- F-interval: $\left(\frac{\phi_{L(\alpha / 2)}}{\hat{p}_{X} \phi_{L(\alpha / 2)}+\hat{p}_{X^{c}}}, \frac{\phi_{U(\alpha / 2)}}{\hat{p}_{X} \phi_{U(\alpha / 2)}+\hat{p}_{X^{c}}}\right)$

with

$$
\begin{aligned}
\phi_{L(\alpha / 2)} & =\frac{R_{X}}{\tilde{R}_{X^{c}}} F_{\left(2 R_{X}^{2} / V_{X}, 2 \tilde{R}_{X^{c}}^{2} / \tilde{V}_{X^{c}}\right)}^{-1}(\alpha / 2) ; \\
\phi_{U(\alpha / 2)} & =\frac{\tilde{R}_{X}}{R_{X^{c}}} F_{\left(2 \tilde{R}_{X}^{2} / \tilde{V}_{X}, 2 R_{X^{c}}^{2} / V_{X^{c}}\right)}^{-1}(\alpha / 2) ;
\end{aligned}
$$

- Normal Interval: $\left(\frac{\tilde{R}_{X}}{\tilde{R}_{\Omega}} \pm Z_{\alpha / 2} \frac{\tilde{R}_{X} \tilde{R}_{X^{c}}}{\tilde{R}_{\Omega}^{2}} \sqrt{\hat{p}_{X^{c}}\left(\frac{\tilde{V}_{X}}{\tilde{R}_{X}^{2}}+\frac{\tilde{V}_{X^{c}}}{\tilde{R}_{X^{c}}^{2}}\right)}\right)$;

where

$$
\begin{aligned}
& \tilde{R}_{X}=\sum_{j=1}^{J} w_{j} \frac{d_{X j}+\frac{1}{J}}{n_{X j}}, \tilde{R}_{X^{c}}=\sum_{j=1}^{n} w_{j} \frac{d_{X^{c} j}+\frac{1}{J}}{n_{X^{c} j}} \\
& \tilde{V}_{X}=\sum_{j=1}^{J} w_{j}^{2} \frac{d_{X j}+\frac{1}{J}}{n_{X j}^{2}}, \tilde{V}_{X^{c}}=\sum_{j=1}^{n} w_{j}^{2} \frac{d_{X^{c} j}+\frac{1}{J}}{n_{X^{c} j}^{2}} ; \tilde{\rho}_{X, \Omega}=\sum_{j=1}^{J} w_{j}^{2} \frac{d_{X j}+\frac{1}{J}}{n_{X j} n_{\Omega j}} .
\end{aligned}
$$

For the normal-approximation based intervals, if the lower limit is negative, we replace it by 0 . Also note that the correction fraction added to the counts $d_{X j}$ and $d_{X^{c} j}$ does not make any significant difference numerically. It merely avoids the zero rates.

Each of the Tables 1-3 gives the ratio of age-adjusted rates, $\frac{\tilde{R}_{X}}{R_{\Omega}}$, the estimate, $\hat{p}_{X}$, of the ratio of population for region $X$ to that of the US, the empirical coverage probabilities, and the width of the $95 \%$ intervals for the two intervals, namely, the $F$ - and normal-approximation based CIs. Because of the space, we only report the selected states in these tables and the full tables are available from the authors. The results show that the two CIs perform reasonably close. For the tongue cancer, all intervals have higher coverage probabilities and larger 
Table 1: Performance of the Derived Confidence Intervals based on the 2004 Tongue Cancer Mortality Data. The empirical coverage probabilities and the widths of the intervals were based on 10,000 simulations.

\begin{tabular}{|c|c|c|c|c|c|c|}
\hline \multirow[b]{2}{*}{ State } & \multirow[b]{2}{*}{$\begin{array}{l}\text { Rate Ratio } \\
\text { with US }\end{array}$} & \multirow[b]{2}{*}{$\begin{array}{l}\text { Overlap } \\
\text { ratio }\left(p_{X}\right)\end{array}$} & \multicolumn{2}{|c|}{ Empirical Coverage Prob. } & \multicolumn{2}{|c|}{ Average Width } \\
\hline & & & $F$-based & Normal-based & $F$-based & Normal-based \\
\hline California & 1.094 & 0.122 & 0.956 & 0.963 & 0.270 & 0.286 \\
\hline Colorado & 0.984 & 0.016 & 0.957 & 0.952 & 0.821 & 0.802 \\
\hline Connecticut & 0.892 & 0.012 & 0.964 & 0.955 & 0.797 & 0.771 \\
\hline Delaware & 0.900 & 0.003 & 0.979 & 0.961 & 1.790 & 1.706 \\
\hline Washington DC & 2.073 & 0.002 & 0.968 & 0.957 & 3.399 & 3.257 \\
\hline Florida & 1.281 & 0.059 & 0.956 & 0.960 & 0.388 & 0.391 \\
\hline Georgia & 0.921 & 0.030 & 0.959 & 0.955 & 0.555 & 0.548 \\
\hline Hawaii & 0.463 & 0.004 & 0.973 & 0.971 & 1.054 & 1.001 \\
\hline Idaho & 1.441 & 0.005 & 0.964 & 0.955 & 1.751 & 1.686 \\
\hline Illinois & 1.019 & 0.043 & 0.955 & 0.955 & 0.450 & 0.450 \\
\hline Indiana & 0.886 & 0.021 & 0.958 & 0.953 & 0.601 & 0.588 \\
\hline Iowa & 0.883 & 0.010 & 0.965 & 0.958 & 0.838 & 0.807 \\
\hline Kansas & 0.970 & 0.009 & 0.961 & 0.951 & 0.963 & 0.928 \\
\hline Kentucky & 0.872 & 0.014 & 0.962 & 0.957 & 0.738 & 0.715 \\
\hline Louisiana & 1.016 & 0.015 & 0.962 & 0.956 & 0.785 & 0.765 \\
\hline Maine & 1.306 & 0.004 & 0.973 & 0.967 & 1.566 & 1.496 \\
\hline Maryland & 0.817 & 0.019 & 0.958 & 0.951 & 0.624 & 0.609 \\
\hline Massachusetts & 0.932 & 0.022 & 0.961 & 0.957 & 0.601 & 0.589 \\
\hline Michigan & 0.953 & 0.034 & 0.958 & 0.956 & 0.481 & 0.476 \\
\hline Minnesota & 0.906 & 0.017 & 0.963 & 0.956 & 0.691 & 0.674 \\
\hline Mississippi & 0.377 & 0.010 & 0.971 & 0.956 & 0.618 & 0.593 \\
\hline Missouri & 0.945 & 0.020 & 0.959 & 0.955 & 0.641 & 0.626 \\
\hline Montana & 0.737 & 0.003 & 0.980 & 0.977 & 1.514 & 1.441 \\
\hline Nebraska & 0.786 & 0.006 & 0.963 & 0.955 & 1.124 & 1.079 \\
\hline Nevada & 1.214 & 0.008 & 0.963 & 0.955 & 1.230 & 1.188 \\
\hline New Hampshire & 1.087 & 0.004 & 0.969 & 0.962 & 1.560 & 1.496 \\
\hline New Jersey & 1.191 & 0.030 & 0.955 & 0.953 & 0.573 & 0.567 \\
\hline New Mexico & 0.936 & 0.006 & 0.965 & 0.956 & 1.202 & 1.157 \\
\hline New York & 1.129 & 0.066 & 0.953 & 0.956 & 0.363 & 0.369 \\
\hline North Carolina & 0.928 & 0.029 & 0.959 & 0.956 & 0.533 & 0.526 \\
\hline Pennsylvania & 0.841 & 0.042 & 0.954 & 0.954 & 0.384 & 0.383 \\
\hline Texas & 1.044 & 0.077 & 0.954 & 0.955 & 0.356 & 0.365 \\
\hline
\end{tabular}

widths than those for esophagus and lung cancers. Indeed, for the latter two cancers, the empirical coverage probabilities get much closer to 95\%. For large states such as California, Florida, New York, Pennsylvania, and Texas, all intervals perform similarly in terms of interval widths and the coverage probabilities.

\section{Discussion of the Results}

The SEER Program of NCI has implemented the $F$-intervals of Fay (1999) and the modified $F$-interval of Tiwari et al. (2006) in the SEER*STAT software to compare the age-adjusted rates for two nonoverlapping regions. However, as pointed out in Tiwari et al. (2006), there is an emerging need of obtaining confidence interval formulae for comparing the age-adjusted rates of a subregion 
Table 2: Performance of the Derived Confidence Intervals based on the 2004 Esophagus Cancer Mortality Data. The empirical coverage probabilities and the widths of the intervals were based on 10,000 simulations.

\begin{tabular}{|c|c|c|c|c|c|c|}
\hline \multirow[b]{2}{*}{ State } & \multirow[b]{2}{*}{$\begin{array}{l}\text { Rate Ratio } \\
\text { with US }\end{array}$} & \multirow[b]{2}{*}{$\begin{array}{c}\text { Overlap } \\
\text { ratio }\left(p_{X}\right)\end{array}$} & \multicolumn{2}{|c|}{ Empirical Coverage Prob. } & \multicolumn{2}{|c|}{ Average Width } \\
\hline & & & $F$-based & Normal-based & $F$-based & Normal-based \\
\hline California & 0.842 & 0.122 & 0.952 & 0.961 & 0.092 & 0.097 \\
\hline Colorado & 0.980 & 0.016 & 0.953 & 0.951 & 0.299 & 0.296 \\
\hline Connecticut & 0.968 & 0.012 & 0.957 & 0.953 & 0.306 & 0.302 \\
\hline Delaware & 1.591 & 0.003 & 0.956 & 0.951 & 0.826 & 0.805 \\
\hline Washington DC & 1.286 & 0.002 & 0.961 & 0.954 & 0.939 & 0.908 \\
\hline Florida & 0.951 & 0.059 & 0.957 & 0.964 & 0.122 & 0.125 \\
\hline Georgia & 1.044 & 0.030 & 0.951 & 0.951 & 0.221 & 0.222 \\
\hline Hawaii & 0.563 & 0.004 & 0.963 & 0.958 & 0.395 & 0.382 \\
\hline Idaho & 0.864 & 0.005 & 0.956 & 0.949 & 0.493 & 0.480 \\
\hline Illinois & 1.037 & 0.043 & 0.956 & 0.958 & 0.170 & 0.172 \\
\hline Indiana & 1.080 & 0.021 & 0.951 & 0.950 & 0.247 & 0.246 \\
\hline Iowa & 0.976 & 0.010 & 0.953 & 0.949 & 0.324 & 0.319 \\
\hline Kansas & 0.913 & 0.009 & 0.954 & 0.951 & 0.344 & 0.338 \\
\hline Kentucky & 1.009 & 0.014 & 0.953 & 0.951 & 0.291 & 0.288 \\
\hline Louisiana & 1.034 & 0.015 & 0.950 & 0.948 & 0.292 & 0.290 \\
\hline Maine & 1.336 & 0.004 & 0.960 & 0.956 & 0.565 & 0.551 \\
\hline Maryland & 0.951 & 0.019 & 0.955 & 0.954 & 0.251 & 0.249 \\
\hline Massachusetts & 1.167 & 0.022 & 0.952 & 0.951 & 0.246 & 0.245 \\
\hline Michigan & 1.099 & 0.034 & 0.956 & 0.957 & 0.193 & 0.194 \\
\hline Minnesota & 1.016 & 0.017 & 0.953 & 0.951 & 0.268 & 0.266 \\
\hline Mississippi & 0.946 & 0.010 & 0.953 & 0.950 & 0.345 & 0.339 \\
\hline Missouri & 1.044 & 0.020 & 0.952 & 0.951 & 0.246 & 0.245 \\
\hline Montana & 1.062 & 0.003 & 0.956 & 0.947 & 0.632 & 0.613 \\
\hline Nebraska & 0.989 & 0.006 & 0.958 & 0.953 & 0.447 & 0.438 \\
\hline Nevada & 0.941 & 0.008 & 0.957 & 0.953 & 0.404 & 0.397 \\
\hline New Hampshire & 1.193 & 0.004 & 0.957 & 0.952 & 0.580 & 0.566 \\
\hline New Jersey & 0.941 & 0.030 & 0.955 & 0.955 & 0.190 & 0.191 \\
\hline New Mexico & 0.973 & 0.006 & 0.957 & 0.954 & 0.437 & 0.428 \\
\hline New York & 1.019 & 0.066 & 0.950 & 0.956 & 0.130 & 0.133 \\
\hline North Carolina & 1.024 & 0.029 & 0.952 & 0.953 & 0.206 & 0.206 \\
\hline Pennsylvania & 1.160 & 0.042 & 0.953 & 0.957 & 0.166 & 0.167 \\
\hline Texas & 0.875 & 0.077 & 0.954 & 0.957 & 0.125 & 0.129 \\
\hline
\end{tabular}

that is a part of a larger region. This paper fills that gap by providing the needed confidence interval formulae, namely, the $F$-based and the normal-based intervals. It is noticeable that these two intervals depend on the ratio of the population sizes for the subregion and the region, and on their age-adjusted rates. The results in Tables 1-3 show the effect of the size of the overlap in the two populations on the confidence intervals. To avoid the situation where the observed age-adjusted rates are zero, we adopted a corrected version of age-adjusted rates by adding a small constant as in Tiwari et al. (2006).

We note the framework of the normal-approximation method allows the computation for the following two common scenarios in cancer surveillance. The first concerns with a partial overlapping situation. Consider $X$ and $\Omega$ two regions 
Table 3: Performance of the Derived Confidence Intervals based on the 2004 Lung Cancer Mortality Data. The empirical coverage probabilities and the widths of the intervals were based on 10,000 simulations.

\begin{tabular}{|c|c|c|c|c|c|c|}
\hline \multirow[b]{2}{*}{ State } & \multirow[b]{2}{*}{$\begin{array}{c}\text { Rate Ratio } \\
\text { with US }\end{array}$} & \multirow[b]{2}{*}{$\begin{array}{c}\text { Overlap } \\
\text { ratio }\left(p_{X}\right)\end{array}$} & \multicolumn{2}{|c|}{ Empirical Coverage Prob. } & \multicolumn{2}{|c|}{ Average Width } \\
\hline & & & $F$-based & Normal-based & $F$-based & Normal-based \\
\hline California & 0.797 & 0.122 & 0.932 & 0.959 & 0.026 & 0.027 \\
\hline Colorado & 0.740 & 0.016 & 0.952 & 0.953 & 0.075 & 0.076 \\
\hline Connecticut & 0.936 & 0.012 & 0.955 & 0.956 & 0.085 & 0.085 \\
\hline Delaware & 1.234 & 0.003 & 0.948 & 0.946 & 0.203 & 0.201 \\
\hline Washington DC & 0.959 & 0.002 & 0.953 & 0.949 & 0.228 & 0.225 \\
\hline Florida & 1.012 & 0.059 & 0.951 & 0.961 & 0.036 & 0.037 \\
\hline Georgia & 1.101 & 0.030 & 0.948 & 0.950 & 0.065 & 0.066 \\
\hline Hawaii & 0.703 & 0.004 & 0.956 & 0.955 & 0.123 & 0.122 \\
\hline Idaho & 0.772 & 0.005 & 0.953 & 0.951 & 0.133 & 0.132 \\
\hline Illinois & 1.029 & 0.043 & 0.946 & 0.952 & 0.048 & 0.049 \\
\hline Indiana & 1.145 & 0.021 & 0.950 & 0.951 & 0.072 & 0.073 \\
\hline Iowa & 1.009 & 0.010 & 0.952 & 0.952 & 0.094 & 0.093 \\
\hline Kansas & 1.017 & 0.009 & 0.952 & 0.952 & 0.102 & 0.102 \\
\hline Kentucky & 1.448 & 0.014 & 0.953 & 0.954 & 0.099 & 0.099 \\
\hline Louisiana & 1.255 & 0.015 & 0.954 & 0.954 & 0.091 & 0.091 \\
\hline Maine & 1.145 & 0.004 & 0.952 & 0.951 & 0.147 & 0.146 \\
\hline Maryland & 0.998 & 0.019 & 0.948 & 0.949 & 0.074 & 0.074 \\
\hline Massachusetts & 0.980 & 0.022 & 0.953 & 0.955 & 0.064 & 0.065 \\
\hline Michigan & 1.068 & 0.034 & 0.955 & 0.958 & 0.054 & 0.055 \\
\hline Minnesota & 0.881 & 0.017 & 0.948 & 0.949 & 0.071 & 0.072 \\
\hline Mississippi & 1.282 & 0.010 & 0.952 & 0.952 & 0.114 & 0.113 \\
\hline Missouri & 1.224 & 0.020 & 0.950 & 0.952 & 0.075 & 0.076 \\
\hline Montana & 0.921 & 0.003 & 0.953 & 0.950 & 0.164 & 0.162 \\
\hline Nebraska & 0.888 & 0.006 & 0.952 & 0.951 & 0.119 & 0.118 \\
\hline Nevada & 1.075 & 0.008 & 0.950 & 0.950 & 0.122 & 0.122 \\
\hline New Hampshire & 1.041 & 0.004 & 0.957 & 0.955 & 0.153 & 0.152 \\
\hline New Jersey & 0.913 & 0.030 & 0.952 & 0.954 & 0.053 & 0.054 \\
\hline New Mexico & 0.673 & 0.006 & 0.953 & 0.951 & 0.103 & 0.102 \\
\hline New York & 0.862 & 0.066 & 0.952 & 0.961 & 0.034 & 0.035 \\
\hline North Carolina & 1.111 & 0.029 & 0.953 & 0.955 & 0.061 & 0.061 \\
\hline Pennsylvania & 0.998 & 0.042 & 0.949 & 0.954 & 0.044 & 0.044 \\
\hline Texas & 0.977 & 0.077 & 0.948 & 0.952 & 0.037 & 0.039 \\
\hline
\end{tabular}

with a partial overlap, for example $X=\{$ Geogia, North Carolina, South Carolina $\}$ and $\Omega=\{$ North Carolina, Virginia $\}$, with $X \Omega=\{$ North Carolina $\}$. Also, a Taylor series expansion, similar to that in Section 2.2, for the rate-ratio

$$
\frac{R_{X}}{R_{\Omega}} \doteq \frac{p_{X \Omega} R_{X \Omega}+p_{X \Omega^{c}} R_{X \Omega^{c}}}{q_{X \Omega} R_{X \Omega}+q_{X^{c} \Omega} R_{X^{c} \Omega^{c}}}
$$

can be used to obtain an approximate normal CI. Here, $p_{X \Omega}=n_{X \Omega} / n_{X}, p_{X \Omega^{c}}=$ $n_{X \Omega^{c}} / n_{X}, q_{X \Omega}=n_{X \Omega} / n_{\Omega}, q_{X^{c} \Omega}=n_{X^{c} \Omega} / n_{\Omega}$. The second scenario concerns with comparing two age-adjusted incidence rate ratios, namely (i) $R_{X W}^{(L)} / R_{X W}^{(T)}$ with $R_{X B}^{(L)} / R_{X B}^{(T)}$, and (ii) $R_{X W}^{(L)} / R_{X W}^{(T)}$ with $R_{\Omega W}^{(L)} / R_{\Omega W}^{(T)}$, where $R_{X W}^{(L)}=\sum_{j} w_{j} d_{X W j}^{(L)} / n_{X W j}$ is the age-adjusted incidence rate for white women with localized breast cancer 
in subregion $X \subset \Omega$, and $R_{X W}^{(T)}=\sum_{j} w_{j} d_{X W j}^{(T)} / n_{X W j}$ is the age-adjusted rates for white women with breast cancer with localized, regional, and distant stages combined in $X$, while $R_{X B}^{(L)}=\sum_{j} w_{j} d_{X B j}^{(L)} / n_{X B j}$ and $R_{X B}^{(T)}=\sum_{j} w_{j} d_{X B j}^{(T)} / n_{X B j}$ are the corresponding age-adjusted incidence rates for black women in subregion $X$ respectively. The terms $R_{\Omega W}^{(L)}=\sum_{j} w_{j} d_{\Omega W j}^{(L)} / n_{\Omega W j}$ and $R_{\Omega W}^{(T)}=\sum_{j} w_{j} d_{\Omega W j}^{(T)} / n_{\Omega W j}$ are defined for the age-adjusted incidence rates for white women in Region $\Omega$ similarly. Our focus centers on constructing the confidence intervals based on the following differences of ratios, namely,

$$
D R_{X, W-B}=\frac{R_{X W}^{(L)}}{R_{X W}^{(T)}}-\frac{R_{X B}^{(L)}}{R_{X B}^{(T)}}, \text { and } D R_{X-\Omega, W}=\frac{R_{X W}^{(L)}}{R_{X W}^{(T)}}-\frac{R_{\Omega W}^{(L)}}{R_{\Omega W}^{(T)}} .
$$

Indeed, the approximate $(1-\alpha) \times 100 \%$ confidence intervals based on $D R_{X, W-B}$ and $D R_{X-\Omega, W}$ are $D R_{X, W-B} \mp Z_{\alpha / 2} \times\left(\widehat{V}\left(D R_{X, W-B}\right)\right)^{1 / 2}$ and $D R_{X-\Omega, W} \mp Z_{\alpha / 2} \times$ $\left(\widehat{V}\left(D R_{X-\Omega, W}\right)\right)^{1 / 2}$, respectively, where $\widehat{V}\left(D R_{X, W-B}\right)$ and $\widehat{V}\left(D R_{X-\Omega, W}\right)$ are the variance estimates of $D R_{X-\Omega, W}$ and $D R_{X-\Omega, W}$ given in the Appendix. As an application, we computed the $95 \%$ confidence interval for $D R_{X, W-B}$, the difference of the age-adjusted rate ratio for white women with localized breast cancer to all stages combined with that for the women from the SEER-9 database (year 2003). The observed difference was $10.87 \%$ with the $95 \%$ CI being $(8.32 \%, 13.41 \%)$, demonstrating racial disparities as the rate-ratio for the whites were significantly different from the black women. On the other hand, an application of $D R_{X-\Omega, W}$ was made to compare the rate-ratio for white women in Iowa with that for all white women in the SEER-9 database. The calculated difference was $1.01 \%$ with the $95 \%$ CI being $(-1.20 \%, 3.22 \%)$, revealing no significant differences.

\section{Conclusion}

We derived confidence intervals based on $\mathrm{F}$ and normal approximations for the ratio of age-adjusted rates for a subregion to a parent region containing the subregion. Through simulations, we showed that all the proposed intervals performed well, in terms of retaining the nominal coverage. Our work fills the gap of non-availability of methods that compare the ratio of age-adjusted rates for two overlapping regions. We will suggest that these formulae be implemented in the NCI SEER*STAT software for public use.

\section{References}

Fay, M.P. (1999). Approximate Confidence Intervals for Rate Ratios from Directly Standardized Rates with Sparse Data. Communications in Statistics, Theory and Methods 28, 2141-2160. 
Tiwari, R.C., Clegg, L. and Zou, Z. (2006) Efficient interval estimation for age-adjusted cancer rates. Statistical Methods in Medical Research 15, 547-569.

Pickle, L.W. and White, A.A. (1995). Effects of the choice of age-adjustment method on maps of death rates. Statistics in Medicine 14, 615-627.

\section{Appendix: Technical Detail}

Using the delta method and applying the results derived in Section 2.1, we obtain the estimates of the variance expressions for $D R_{X, W-B}$ and $D R_{X-\Omega, W}$ as follows:

$$
\begin{aligned}
& \widehat{V}\left(D R_{X, W-B}\right)= \\
& \frac{1}{\left(R_{X W}^{(T)}\right)^{4}}\left\{\left(R_{X W}^{(T)}\right)^{2} \widehat{V}\left(R_{X W}^{(L)}\right)+\left(R_{X W}^{(L)}\right)^{2} \widehat{V}\left(R_{X W}^{(T)}\right)-2 R_{X W}^{(T)} R_{X W}^{(L)} \widehat{\operatorname{Cov}}\left(R_{X W}^{(L)}, R_{X W}^{(T)}\right)\right\} \\
& +\frac{1}{\left(R_{X B}^{(T)}\right)^{4}}\left\{\left(R_{X B}^{(T)}\right)^{2} \widehat{V}\left(R_{X B}^{(L)}\right)+\left(R_{X B}^{(L)}\right)^{2} \widehat{V}\left(R_{X B}^{(T)}\right)-2 R_{X B}^{(T)} R_{X B}^{(L)} \widehat{\operatorname{Cov}}\left(R_{X B}^{(L)}, R_{X B}^{(T)}\right)\right\}
\end{aligned}
$$

with $\widehat{\operatorname{Cov}}\left(R_{X W}^{(L)}, R_{X W}^{(T)}\right)=\sum_{j} w_{j}^{2} d_{X W j}^{(L)} / n_{X W j}^{2}$ and $\widehat{\operatorname{Cov}}\left(R_{X B}^{(L)}, R_{X B}^{(T)}\right)=\sum_{j} w_{j}^{2} d_{X B j}^{(L)} / n_{X B j}^{2}$, and

$$
\begin{aligned}
& \widehat{V}\left(D R_{X-\Omega, W}\right)= \\
& \frac{1}{\left(R_{X W}^{(T)}\right)^{4}}\left\{\left(R_{X W}^{(T)}\right)^{2} \widehat{V}\left(R_{X W}^{(L)}\right)+\left(R_{X W}^{(L)}\right)^{2} \widehat{V}\left(R_{X W}^{(T)}\right)-2 R_{X W}^{(T)} R_{X W}^{(L)} \widehat{\operatorname{Cov}}\left(R_{X W}^{(L)}, R_{X W}^{(T)}\right)\right\} \\
& +\frac{1}{\left(R_{\Omega W}^{(T)}\right)^{4}}\left\{\left(R_{\Omega W}^{(T)}\right)^{2} \widehat{V}\left(R_{\Omega W}^{(L)}\right)+\left(R_{\Omega W}^{(L)}\right)^{2} \widehat{V}\left(R_{\Omega W}^{(T)}\right)-2 R_{\Omega W}^{(T)} R_{\Omega W}^{(L)} \widehat{\operatorname{Cov}}\left(R_{\Omega W}^{(L)}, R_{\Omega W}^{(T)}\right)\right\} \\
& -\frac{2}{\left(R_{X W}^{(T)} R_{\Omega W}^{(T)}\right)^{2}}\left\{R_{X W}^{(T)} R_{\Omega W}^{(T)} \widehat{\operatorname{Cov}}\left(R_{X W}^{(L)}, R_{\Omega W}^{(L)}\right)+R_{X W}^{(L)} R_{\Omega W}^{(L)} \widehat{\operatorname{Cov}}\left(R_{X W}^{(T)}, R_{\Omega W}^{(T)}\right)\right. \\
& \left.-R_{X W}^{(L)} R_{\Omega W}^{(T)} \widehat{\operatorname{Cov}}\left(R_{X W}^{(T)}, R_{\Omega W}^{(L)}\right)-R_{X W}^{(T)} R_{\Omega W}^{(L)} \widehat{\operatorname{Cov}}\left(R_{X W}^{(L)}, R_{\Omega W}^{(T)}\right)\right\}
\end{aligned}
$$

with $\widehat{\operatorname{Cov}}\left(R_{\Omega W}^{(L)}, R_{\Omega W}^{(T)}\right)=\sum_{j} w_{j}^{2} d_{\Omega W j}^{(L)} / n_{\Omega W j}^{2}$ and $\widehat{\operatorname{Cov}}\left(R_{X W}^{(L)}, R_{\Omega W}^{(L)}\right)=\widehat{\operatorname{Cov}}\left(R_{X W}^{(T)}, R_{\Omega W}^{(T)}\right)=$ $\widehat{\operatorname{Cov}}\left(R_{X W}^{(T)}, R_{\Omega W}^{(L)}\right)=\widehat{\operatorname{Cov}}\left(R_{X W}^{(L)}, R_{\Omega W}^{(T)}\right)=\sum_{j} w_{j}^{2} d_{X W j}^{(L)} /\left(n_{X W j} \times n_{\Omega W j}\right)$, and $\widehat{\operatorname{Cov}}\left(R_{X W}^{(L)}, R_{X W}^{(T)}\right)$ defined earlier. 
Ram C. Tiwari

Food and Drug Administration

Center for Drug Evaluation \& Research, FDA

10903 New Hampshire Ave.

WO Bldg. 21, Rm. 3524

Silver Spring, MD, 20993-0002, USA

ram.tiwari@fda.hhs.gov

Yi Li

Harvard School of Public Health

44 Binney Street, Boston MA 02115

yili@jimmy.harvard.edu

Zhaohui Zou

Information Management Services

12501 Prosperity Dr, Suite 200

Silver Spring, MD 20904, USA

ZouJ@imsweb.com 